\title{
THE CUNTZ SEMIGROUP, A RIESZ TYPE INTERPOLATION PROPERTY, COMPARISON AND THE IDEAL PROPERTY
}

\author{
Cornel Pasnicu and Francesc Perera
}

\begin{abstract}
We define a Riesz type interpolation property for the Cuntz semigroup of a $C^{*}$-algebra and prove it is satisfied by the Cuntz semigroup of every $C^{*}$-algebra with the ideal property. Related to this, we obtain two characterizations of the ideal property in terms of the Cuntz semigroup of the $C^{*}$-algebra. Some additional characterizations are proved in the special case of the stable, purely infinite $C^{*}$-algebras, and two of them are expressed in language of the Cuntz semigroup. We introduce a notion of comparison of positive elements for every unital $C^{*}$-algebra that has (normalized) quasitraces. We prove that large classes of $C^{*}$-algebras (including large classes of $A H$ algebras) with the ideal property have this comparison property.
\end{abstract}

2010 Mathematics Subject Classification: Primary: 46L35; Secondary: 46L05.

Key words: $C^{*}$-algebra, the Cuntz semigroup, a Riesz type interpolation property, ideal property, comparison of positive elements, $A H$ algebra.

\section{Introduction}

Elliott's classification program for separable, nuclear $C^{*}$-algebras by discrete invariants including the $K$-theory is one of the most important and successful research areas in Operator Algebras ([9], $[\mathbf{1 0}]$; see also [24]). While it is clear that not all the separable, nuclear $C^{*}$-algebras can be classified, very large classes of $C^{*}$-algebras are known to be classifiable (see, e.g., [24]). Despite the success, counterexamples to Elliott's conjecture in the simple case were found by Rørdam [25] and by Toms ([28] and $[\mathbf{2 9}])$. In [29] Toms used the Cuntz semigroup to distinguish simple, nuclear $C^{*}$-algebras which cannot be distinguished by the conventional Elliott invariant, where the Cuntz semigroup of a $C^{*}$ algebra $A$ is a positively ordered, abelian semigroup whose elements are equivalence classes of positive elements in matrix algebras over $A$ (see Section 2 for details). It then became clear that a further study of the Cuntz semigroup is needed, and that, in fact, it is very important in Elliott's classification program. On the other hand, as pointed out in [10], the understanding of the regularity properties of simple $C^{*}$-algebras, 
including the comparison of positive elements, is essential in the new development of Elliott's program. Extending the notion of comparison of positive elements to classes of non-simple $C^{*}$-algebras - e.g., to the $C^{*}$-algebras with the ideal property - and proving appropriate comparison results for these classes is clearly a necessary and non-trivial thing to do. In this paper we contribute to the study of the ideal property, to the understanding of the structure of the Cuntz semigroup of $C^{*}$-algebras (with this property), and prove a certain type of comparison of positive elements for some classes of $C^{*}$-algebras with the ideal property.

A $C^{*}$-algebra is said to have the ideal property if each of its ideals is generated (as an ideal) by its projections (in this paper, by an ideal we mean a closed, two-sided ideal; the only exception here will be the Pedersen ideal, which is the smallest dense algebraic two-sided ideal in the $C^{*}$-algebra). Note that every simple $C^{*}$-algebra with an approximate unit of projections and every $C^{*}$-algebra of real rank zero [4] have the ideal property. The ideal property has been studied extensively by the first named author (alone or in collaboration), for example in [18], [19], [20], [21] and [13], [14]. The ideal property is important in Elliott's classification program. It appeared first in Ken Stevens' Ph.D. thesis in which he classified by a $K$-theoretical invariant a certain class of (nonsimple) $A I$ algebras with the ideal property. In $[\mathbf{1 8}]$ the first named author classified the $A H$ algebras with the ideal property and with slow dimension growth up to a shape equivalence and gave several characterizations of when an arbitrary $A H$ algebra has the ideal property. Recall that a $C^{*}$-algebra $A$ is said to be an $A H$ algebra, if $A$ is the inductive limit $C^{*}$-algebra of:

$$
A_{1} \stackrel{\phi_{1,2}}{\longrightarrow} A_{2} \stackrel{\phi_{2,3}}{\longrightarrow} A_{3} \stackrel{\phi_{3,4}}{\longrightarrow} \cdots \stackrel{\phi_{n-1, n}}{\longrightarrow} A_{n} \stackrel{\phi_{n, n+1}}{\longrightarrow} \cdots
$$

with $A_{n}=\bigoplus_{i=1}^{t_{n}} P_{n, i} M_{[n, i]}\left(C\left(X_{n, i}\right)\right) P_{n, i}$, where the local spectra $X_{n, i}$ are finite, connected $C W$ complexes, $t_{n}$ and $[n, i]$ are strictly positive integers, and each $P_{n, i}$ is a projection of $M_{[n, i]}\left(C\left(X_{n, i}\right)\right)$. In [13] and [14], jointly with Gong, Jiang and Li, the first named author proved a reduction theorem saying that every $A H$ algebra with the ideal property and with the dimensions of the local spectra uniformly bounded (i.e., with no dimension growth) can be written as an $A H$ algebra with the ideal property with (special) local spectra of dimensions $\leq 3$. This result generalizes similar and strong reduction theorems for real rank zero $A H$ algebras - proved by Dădărlat [8] and Gong [11] - and also for simple $A H$ algebras - proved by Gong [12] - which have been major steps in the classification of the corresponding classes of $A H$ algebras. Also, in [18] and $[\mathbf{1 9}]$, the first named author proved several nonstable $K$-theoretical 
results for a large class of $C^{*}$-algebras with the ideal property. Indeed, if $A$ is an $A H$ algebra with the ideal property and with slow dimension growth, it is proved in [18] that $A$ has stable rank one (that means, in the unital case, that the set of the invertible elements in $A$ is dense in $A$ ), that $K_{0}(A)$ is weakly unperforated in the sense of Elliott and is also a Riesz group ([18] and [19]) and that the strict comparability of the projections in $A$ is determined by the tracial states of $A$, when $A$ is unital [18]. Also, jointly with Rørdam, the first named author proved in [20] that the ideal property is not preserved by taking minimal tensor products (even in the separable case). A characterization of the ideal property in the separable, purely infinite case is given by the first named author jointly with Rørdam in [21], in terms of the Jacobson topology of the primitive spectrum of the $C^{*}$-algebra. The class of purely infinite $C^{*}$-algebras has been introduced by Kirchberg and Rørdam in [17], extending the definition in the simple case given by Cuntz [7]. A $C^{*}$ algebra $A$ is said to be purely infinite if $A$ has no characters (or, equivalently, no non-zero abelian quotients) and if for every $a, b \in A^{+}$such that $a \in \overline{A b A}$ (the ideal of $A$ generated by $b$ ), it follows that there is a sequence $\left\{x_{n}\right\}$ of elements in $A$ such that $a=\lim _{n \rightarrow \infty} x_{n}^{*} b x_{n}$ [17]. The study of purely infinite $C^{*}$-algebras was motivated by Kirchberg's classification of the separable, nuclear $C^{*}$-algebras that tensorially absorb the Cuntz algebra $\mathcal{O}_{\infty}$ up to stable isomorphism by an ideal related $K K$-theory.

The paper is divided into three sections. In Section 2 we remind the reader some relevant definitions and notation - including the definition of the Cuntz semigroup - and define a Riesz type interpolation property for the Cuntz semigroup $W(A)$ of a $C^{*}$-algebra $A$ (Definition 2.1). We prove that $W(A)$ has this property, whenever $A$ is a $C^{*}$-algebra the ideal property (Theorem 2.2). Related to this, we obtain two characterizations of the ideal property in terms of the Cuntz semigroup of the $C^{*}$-algebra (Theorem 2.4). Some additional characterizations are proved in the special case of the stable, purely infinite $C^{*}$-algebras, and two of them are expressed in language of the Cuntz semigroup of the algebra (Theorem 2.5). In Section 3, after reminding the reader some definitions, notation and results, we introduce a notion of comparison of positive elements for every unital $C^{*}$-algebra that has (normalized) quasitraces (Definition 3.1). We prove that large classes of $C^{*}$-algebras (including large classes of $A H$ algebras) with the ideal property have this comparison property (Theorems 3.6, 3.7 and 3.8).

The symbol $\otimes$ will mean the minimal tensor product of $C^{*}$-algebras. 


\section{A Riesz type interpolation property for the Cuntz semigroup and the ideal property}

We start by recalling mainly some definitions and notation (see also [2]). If $A$ is a $C^{*}$-algebra and $a, b \in A^{+}$, then we write $a \precsim b$ if there is a sequence $\left\{x_{n}\right\}$ of elements of $A$ such that $a=\lim _{n \rightarrow \infty} x_{n} b x_{n}^{*}$. This relation can be extended to the (local) $C^{*}$-algebra $M_{\infty}(A)$ defined as the algebraic inductive limit of $M_{n}(A)$ via the inclusion mappings $M_{n}(A) \hookrightarrow M_{n+1}(A)$ given by $x \mapsto x \oplus 0$. Let $M_{\infty}(A)^{+}$denote the set of positive elements of $M_{\infty}(A)$. If $a, b \in M_{\infty}(A)^{+}$, we write $a \precsim b$ provided that $a \precsim b$ in $M_{n}(A)$ for some $n$ such that $a, b \in M_{n}(A)$. (If we view $a$ and $b$ in two different sized matrices over $A$, the above is equivalent to having $a=\lim _{n \rightarrow \infty} x_{n} b x_{n}^{*}$ where the $x_{n}$ are suitable rectangular matrices.) If both $a \precsim b$ and $b \precsim a$, we will write $a \sim b$ and will call $a$ and $b$ Cuntz equivalent (see $[\mathbf{6}]$ ). We shall denote $W(A)=M_{\infty}(A)^{+} / \sim$, and $\langle a\rangle \in W(A)$ will denote the Cuntz equivalence class of an element $a$ of $M_{\infty}(A)^{+}$(so that $W(A)=\left\{\langle a\rangle: a \in M_{\infty}(A)^{+}\right\}$). Then $W(A)$ is a positively ordered abelian semigroup when equipped with the relations:

$$
\langle a\rangle+\langle b\rangle=\langle a \oplus b\rangle,\langle a\rangle \leq\langle b\rangle \Leftrightarrow a \precsim b, \quad a, b \in M_{\infty}(A)^{+} .
$$

We shall refer to $W(A)$ as the Cuntz semigroup of $A$. One shortcoming of this construction is that this semigroup fails to be continuous with respect to sequential inductive limits. This was remedied in [5] by constructing an ordered semigroup, termed $C u(A)$, in terms of countably generated Hilbert modules. This new object turned out to be intimately related to $W(A)$, in that $C u(A)$ is order isomorphic to $W(A \otimes \mathcal{K})$, where $\mathcal{K}$ is the $C^{*}$-algebra of the compact operators on $\ell^{2}(\mathbb{N})[\mathbf{5}]$. The semigroup $C u(A)$ is, as opposed to $W(A)$, closed under (order-theoretic) suprema of increasing sequences and, in significant cases, can be regarded as a completion of $W(A)$ (see [5], [1] ). Moreover, every element in $C u(A)$ is a supremum of a rapidly increasing sequence. More precisely, we write $x \ll y$ to mean that whenever $y \leq \sup y_{n}$ for an increasing sequence $\left\{y_{n}\right\}$, there is $m$ such that $x \leq y_{m}$. A sequence $\left\{x_{n}\right\}$ is rapidly increasing if $x_{n} \ll x_{n+1}$ for all $n$. It is shown in [5] that, for any positive $a$, the sequence $\left\{\left\langle(a-1 / n)_{+}\right\rangle\right\}$is rapidly increasing (and with supremum $\langle a\rangle)$.

If $A$ is a (local) $C^{*}$-algebra, then denote the set of projections in $A$ by $\mathcal{P}(A):=\left\{p \in A: p=p^{2}=p^{*}\right\}$.

For each $a \in A^{+}$and for each $\varepsilon>0$, we shall write $(a-\varepsilon)_{+}$for the positive element of $A$ given by $h_{\varepsilon}(a)$, where $h_{\varepsilon}(t)=\max \{t-\varepsilon, 0\}$. 
Definition 2.1. Let $A$ be a $C^{*}$-algebra. We say that the Cuntz semigroup $W(A)$ has the weak Riesz interpolation by projections property if for all $a_{i}, b_{i} \in M_{\infty}(A)^{+}$such that $\left\langle a_{i}\right\rangle \leq\left\langle b_{j}\right\rangle$ (in $W(A)$ ), $1 \leq i, j \leq 2$ and for every $\varepsilon>0$, there exist a projection $p \in \mathcal{P}\left(M_{\infty}(A)\right)$ and $m \in \mathbb{N}$ such that $\left\langle\left(a_{i}-\varepsilon\right)_{+}\right\rangle \leq\langle p\rangle \leq m\left\langle b_{j}\right\rangle, 1 \leq i, j \leq 2$ (in $W(A)$ ).

This property just defined is of course related to the property of Riesz interpolation by general positive elements. This can be achieved in the real rank zero setting [22], as well as for simple stable algebras that absorb the Jiang-Su algebra [27].

We want to prove the following result:

Theorem 2.2. Let $A$ be a $C^{*}$-algebra with the ideal property. Then $W(A)$ has the weak Riesz interpolation by projections property.

The proof of the above theorem will use the following, of which part (ii) is known and essentially contained in [17]; we include it here just for convenience.

Lemma 2.3. Let $A$ be a $C^{*}$-algebra, let $I$ be an ideal of $A$ that is generated (as an ideal) by $\mathcal{P}(I)$ and let $a \in A^{+}$.

(i) If $a \in I$, then for every $\varepsilon>0$, there exists $p \in \mathcal{P}\left(M_{\infty}(A)\right)$ such that $(a-\varepsilon)_{+} \precsim p$, where $p$ is a finite direct sum of projections of $I$.

(ii) For every $q \in \mathcal{P}(\overline{A a A})$, there exists $n \in \mathbb{N}$ such that $q \precsim a \otimes 1_{n}$.

Proof: (i) Since $I$ is generated by its projections and the element $(a-\varepsilon)_{+}$ belongs to the Pedersen ideal of $I$, we can write $(a-\varepsilon)_{+}=\sum_{i=1}^{k} x_{i} p_{i} y_{i}$, where $x_{i}, y_{i} \in A$ and $p_{i}$ are projections from $I$. Next,

$$
\begin{aligned}
(a-\varepsilon)_{+} & =\sum_{i=1}^{k} x_{i} p_{i} y_{i}=\sum_{i=1}^{k} y_{i}^{*} p_{i} x_{i}^{*} \\
& =\frac{1}{2} \sum_{i=1}^{k}\left(x_{i} p_{i} y_{i}+y_{i}^{*} p_{i} x_{i}^{*}\right) \leq \frac{1}{2} \sum_{i=1}^{k}\left(x_{i} p_{i} x_{i}^{*}+y_{i}^{*} p_{i} y_{i}\right),
\end{aligned}
$$

from which the conclusion follows using [17, Lemma 2.5(ii) and Lemma 2.8(ii)].

(ii) Fix some $0<\varepsilon<1$. Since $q \in \mathcal{P}(\overline{A a A})$, using [17, Proposition 2.7(v)], we get for this $\varepsilon>0$ that there exists some $n \in \mathbb{N}$ such that:

$$
q \sim(q-\varepsilon)_{+} \precsim a \otimes 1_{n},
$$

as wanted. 
Proof of Theorem 2.2: Let $a_{i}, b_{i} \in M_{\infty}(A)^{+}$such that $\left\langle a_{i}\right\rangle \leq\left\langle b_{j}\right\rangle, 1 \leq$ $i, j \leq 2$. We may suppose that $a_{i}, b_{i} \in A^{+}, 1 \leq i \leq 2$. Let $\varepsilon>0$. Note that

$$
\left\langle a_{i}\right\rangle \leq\left\langle a_{1}+a_{2}\right\rangle
$$

for $i=1,2$. Then, by [23], for our $\varepsilon>0$, there exists $\delta>0$ such that:

$$
\left\langle\left(a_{i}-\varepsilon\right)_{+}\right\rangle \leq\left\langle(c-\delta)_{+}\right\rangle, \quad 1 \leq i \leq 2,
$$

where $c=a_{1}+a_{2}$. Since $\left\langle a_{i}\right\rangle \leq\left\langle b_{j}\right\rangle, 1 \leq i, j \leq 2$, we have that $c \in \overline{A b_{j} A}$, $1 \leq j \leq 2$, i.e. $c \in I:=\overline{A b_{1} A} \cap \overline{A b_{2} A}$. Note that since $A$ has the ideal property and $I$ is an ideal of $A$, it follows that $I$ is generated (as an ideal) by $\mathcal{P}(I)$. Then, by Lemma 2.3 , it follows that for our $\delta>0$ there exists $p \in \mathcal{P}\left(M_{\infty}(A)\right)$ such that $p$ is a finite direct sum of projections of $I$ and there exists $m \in \mathbb{N}$ such that:

$$
\left\langle(c-\delta)_{+}\right\rangle \leq\langle p\rangle \leq m\left\langle b_{j}\right\rangle, \quad 1 \leq j \leq 2 .
$$

Finally, (1) and (2) imply that:

$$
\left\langle\left(a_{i}-\varepsilon\right)_{+}\right\rangle \leq\langle p\rangle \leq m\left\langle b_{j}\right\rangle, \quad 1 \leq i, j \leq 2,
$$

which ends the proof.

We now prove a theorem inspired by the proof of Theorem 2.2 and that gives two characterizations of the ideal property in terms of the Cuntz semigroup of the $C^{*}$-algebra. In particular, it implies Theorem 2.2.

Theorem 2.4. Let $A$ be a $C^{*}$-algebra. Then, the following are equivalent:

(i) A has the ideal property.

(ii) For all $a_{i}, b_{i} \in A^{+}$such that $\left\langle a_{i}\right\rangle \leq\left\langle b_{j}\right\rangle, 1 \leq i, j \leq 2$ and for every $\varepsilon>0$, there exist a projection $p \in \mathcal{P}\left(M_{\infty}(A)\right)$ and $m \in \mathbb{N}$ such that $\left\langle\left(a_{i}-\varepsilon\right)_{+}\right\rangle \leq\langle p\rangle \leq m\left\langle b_{j}\right\rangle, 1 \leq i, j \leq 2$ and $p$ is a finite direct sum of projections of $A$.

(iii) For every $a \in A^{+}$and every $\varepsilon>0$, there are a projection $p \in$ $\mathcal{P}\left(M_{\infty}(A)\right)$ and $m \in \mathbb{N}$ such that $\left\langle(a-\varepsilon)_{+}\right\rangle \leq\langle p\rangle \leq m\langle a\rangle$ and $p$ is a finite direct sum of projections of $A$.

Proof: (i) $\Rightarrow$ (ii) It follows from the proof of Theorem 2.2.

(ii) $\Rightarrow$ (iii) Let $a$ be an arbitrary element of $A^{+}$. Then choose $a_{i}=b_{i}=a$, $1 \leq i \leq 2$ and use (ii).

(iii) $\Rightarrow$ (i) Let $I$ be an ideal of $A, a \in I \cap A^{+}$and $\varepsilon>0$. Then, by (iii), there exist $n \in \mathbb{N}, p_{1}, p_{2}, \ldots, p_{n} \in \mathcal{P}(A)$ and there exists $m \in \mathbb{N}$ such 
that:

$$
\left\langle(a-\varepsilon)_{+}\right\rangle \leq\left\langle\oplus_{k=1}^{n} p_{k}\right\rangle \leq m\langle a\rangle .
$$

Increasing $m$ and then $n$, if necessary, (defining the new $p_{k}^{\prime} s$ to be 0 ) we may suppose that $m=n$ in (3). Working in $M_{n}(A)$, it is easy to see that the first inequality in (3) implies that:

$$
(a-\varepsilon)_{+} \in \overline{A p_{1} A+A p_{2} A+\cdots+A p_{n} A}
$$

while the second inequality in (3) implies immediately that:

$$
p_{k} \in \overline{A a A}, \quad 1 \leq k \leq n .
$$

Observe that (4) and (5) imply that $(a-\varepsilon)+$ belongs to the ideal of $A$ generated by $\mathcal{P}(\overline{A a A}) \subseteq \mathcal{P}(I)$. Therefore, $a=\lim _{\varepsilon \rightarrow 0}(a-\varepsilon)_{+}$belongs to the ideal of $A$ generated by $\mathcal{P}(I)$ and hence, because each element of $I$ is a linear combination of four positive elements of $I, I$ is generated as an ideal of $A$ by $\mathcal{P}(I)$. Since $I$ is an arbitrary ideal of $A$, this proves that $A$ has the ideal property.

The result below gives some additional characterizations of the ideal property in the case of stable, purely infinite $C^{*}$-algebras. Two of these characterizations are in terms of the Cuntz semigroup of the $C^{*}$-algebra. As already mentioned in the introduction, a $C^{*}$-algebra $A$ is termed purely infinite if $A$ does not have non-zero abelian quotients and $a \precsim b$ whenever $a \in \overline{A b A}$ (see [17]). Recall also that a positive, non-zero element $a$ of a $C^{*}$-algebra $A$ is said to be properly infinite if $a \oplus a \precsim a \oplus 0$ in $M_{2}(A)$. It was shown in [17, Theorem 4.16] that a $C^{*}$-algebra $A$ is purely infinite if and only if all non-zero positive elements are properly infinite.

Theorem 2.5. Let $A$ be a purely infinite, stable $C^{*}$-algebra. Then, the following are equivalent:

(i) A has the ideal property.

(ii) For every $a \in A^{+}$, there exists a sequence $\left\{p_{n}\right\}$ of projections in $A$ such that $\langle a\rangle=\sup _{n \in \mathbb{N}}\left\langle p_{n}\right\rangle$ (in $\left.W(A)\right)$.

(iii) For every $a \in A^{+}$, there exists a sequence $\left\{q_{n}\right\}$ of projections in $A$ such that $\left\{\left\langle q_{n}\right\rangle\right\}$ is increasing in $W(A)$ and $\langle a\rangle=\sup _{n \in \mathbb{N}}\left\langle q_{n}\right\rangle$ (in $W(A)$ ).

(iv) For all $a \in A^{+}$, we have that $\overline{A a A}=\overline{\cup_{n \geq 1} I_{n}}$, where $\left\{I_{n}\right\}$ is an increasing sequence of ideals of $A$ and each $I_{n}$ is generated (as an ideal) by a single projection. 
The proof of the above theorem will use the following:

Proposition 2.6. Let $A$ be a $C^{*}$-algebra with the ideal property and let $a \in A^{+}$.

(i) If a is either properly infinite or zero, then there is a sequence $\left\{p_{n}\right\}$ of projections in $M_{\infty}(A)$ such that $\langle a\rangle=\sup _{n \in \mathbb{N}}\left\langle p_{n}\right\rangle$ (in $\left.W(A)\right)$.

(ii) If $(a-\varepsilon)_{+}$is either properly infinite or zero for every $\varepsilon>0$, then there is a sequence $\left\{q_{n}\right\}$ of projections in $M_{\infty}(A)$ such that $\left\{\left\langle q_{n}\right\rangle\right\}$ is an increasing sequence in $W(A)$ and $\langle a\rangle=\sup _{n \in \mathbb{N}}\left\langle q_{n}\right\rangle$ (in $\left.W(A)\right)$.

Proof: (i) Let $\left\{\varepsilon_{n}\right\}$ be a strictly decreasing sequence of strictly positive numbers such that $\lim _{n \rightarrow \infty} \varepsilon_{n}=0$. Since $A$ has the ideal property, Theorem 2.4 implies that there exists a sequence $\left\{p_{n}\right\}$ of projections of $M_{\infty}(A)$ such that:

$$
\left\langle\left(a-\varepsilon_{n}\right)_{+}\right\rangle \leq\left\langle p_{n}\right\rangle \leq\langle a\rangle, \text { for all } n \in \mathbb{N}
$$

(we also used the fact that $m\langle a\rangle=\langle a\rangle$, for every $m \in \mathbb{N}$, since $a$ is either properly infinite or zero). Let us prove now that $\sup _{n \in \mathbb{N}}\left\langle p_{n}\right\rangle=\langle a\rangle$. For this, observe first that by (6) we have $\left\langle p_{n}\right\rangle \leq\langle a\rangle$ for every $n \in \mathbb{N}$. Now, let $x \in W(A)$ be such that $\left\langle p_{n}\right\rangle \leq x$, for every $n \in \mathbb{N}$. Then, (6) implies that $\left\langle\left(a-\varepsilon_{n}\right)_{+}\right\rangle \leq x$, for every $n \in \mathbb{N}$. Therefore, since also $\left\{\varepsilon_{n}\right\}$ is a strictly decreasing sequence of strictly positive numbers and $\lim _{n \rightarrow \infty} \varepsilon_{n}=0$, we have:

$$
\langle a\rangle=\sup _{n \in \mathbb{N}}\left\langle\left(a-\varepsilon_{n}\right)_{+}\right\rangle \leq x .
$$

This ends the proof of the equality $\sup _{n \in \mathbb{N}}\left\langle p_{n}\right\rangle=\langle a\rangle$.

(ii) Let $\left\{\varepsilon_{n}\right\}$ be a strictly decreasing sequence of strictly positive numbers such that $\lim _{n \rightarrow \infty} \varepsilon_{n}=0$. Then, since $\varepsilon_{n}-\varepsilon_{n+1}>0$ and

$$
\left(a-\varepsilon_{n}\right)_{+}=\left(\left(a-\varepsilon_{n+1}\right)_{+}-\left(\varepsilon_{n}-\varepsilon_{n+1}\right)\right)_{+}
$$

for every $n \in \mathbb{N}$, Theorem 2.4 implies that there exists a sequence $\left\{q_{n}\right\}$ of projections of $M_{\infty}(A)$ such that:

$$
\left\langle\left(a-\varepsilon_{n}\right)_{+}\right\rangle \leq\left\langle q_{n}\right\rangle \leq\left\langle\left(a-\varepsilon_{n+1}\right)_{+}\right\rangle \leq\langle a\rangle, \text { for all } n \in \mathbb{N}
$$

(we also used the fact that $m\left\langle\left(a-\varepsilon_{n+1}\right)_{+}\right\rangle=\left\langle\left(a-\varepsilon_{n+1}\right)_{+}\right\rangle$, for every $m, n \in \mathbb{N}$, since, by hypothesis, $\left(a-\varepsilon_{n+1}\right)_{+}$is either properly infinite or zero for all $n \in \mathbb{N}$ ). Clearly, (7) implies that $\left\{\left\langle q_{n}\right\rangle\right\}$ is an increasing sequence in $W(A)$. Finally, let us observe that $\sup _{n \in \mathbb{N}}\left\langle q_{n}\right\rangle=\langle a\rangle$ in $W(A)$, 
since its proof is similar with that of the fact that $\sup _{n \in \mathbb{N}}\left\langle p_{n}\right\rangle=\langle a\rangle$ in $W(A)$ in the above proof of part (i) of this proposition.

Corollary 2.7. Let $A$ be a purely infinite $C^{*}$-algebra with the ideal property and let $a \in A^{+}$. Then, there exists a sequence $\left\{p_{n}\right\}$ of projections in $M_{\infty}(A)$ such that $\left\{\left\langle p_{n}\right\rangle\right\}$ is an increasing sequence in $W(A)$ and $\langle a\rangle=\sup _{n \in \mathbb{N}}\left\langle p_{n}\right\rangle$ (in $\left.W(A)\right)$.

Proof: Since $A$ is purely infinite, [17, Theorem 4.16] implies that every positive element of $A$ is either properly infinite or zero. The proof ends now applying condition (ii) in Proposition 2.6.

Proof of Theorem 2.5: (i) $\Rightarrow$ (iii) It follows immediately from Corollary 2.7 .

(iii) $\Rightarrow$ (ii) The proof of this implication is trivial.

(ii) $\Rightarrow$ (iv) Assume (ii). Let $a \in A^{+}$. Then, by (ii), there exists a sequence $\left\{p_{n}\right\}$ of projections of $A$ such that:

$$
\langle a\rangle=\sup _{n \in \mathbb{N}}\left\langle p_{n}\right\rangle \text {. }
$$

Since $A$ is stable, replacing each $p_{n}$ by a projection in its Murray-von Neumann equivalence class we may suppose that $p_{m} p_{n}=0$ for every $m \neq$ $n, m, n \in \mathbb{N}$. Then, for every $n \in \mathbb{N}$ we have that $p_{1}+p_{2}+\cdots+p_{n} \in \mathcal{P}(A)$ and $p_{n} \leq p_{1}+p_{2}+\cdots+p_{n}$ and hence, using [17, Lemma 2.8(iii) and Lemma 2.9] we have:

(9) $\left\langle p_{n}\right\rangle \leq\left\langle p_{1}+p_{2}+\cdots+p_{n}\right\rangle=\sum_{i=1}^{n}\left\langle p_{k}\right\rangle \leq n\langle a\rangle=\langle a\rangle$, for all $n \in \mathbb{N}$

since $\left\langle p_{k}\right\rangle \leq\langle a\rangle$ for all $k \in \mathbb{N}$ and $a$ is properly infinite or zero (by $[\mathbf{1 7}$, Theorem 4.16]). Define:

$$
q_{n}:=p_{1}+p_{2}+\cdots+p_{n}(\in \mathcal{P}(A)) \text {, for all } n \in \mathbb{N} .
$$

Since $p_{1}+p_{2}+\cdots+p_{n} \leq p_{1}+p_{2}+\cdots+p_{n+1}$, for all $n \in \mathbb{N}$, it follows that $\left\{\left\langle q_{n}\right\rangle\right\}$ is an increasing sequence in $W(A)$. Also, (8) and (9) imply that

$$
\langle a\rangle=\sup _{n \in \mathbb{N}}\left\langle q_{n}\right\rangle
$$

We want to prove that:

$$
\overline{A a A}=\overline{\cup_{n \geq 1} A q_{n} A}\left(=\overline{\cup_{n \geq 1} \overline{A q_{n} A}}\right)
$$


(note that $\left\langle q_{n}\right\rangle \leq\left\langle q_{n+1}\right\rangle$ implies that $\overline{A q_{n} A} \subseteq \overline{A q_{n+1} A}$, for all $n \in \mathbb{N}$ ). Indeed, observe that (10) implies that $\left\langle q_{n}\right\rangle \leq\langle a\rangle$, for all $n \in \mathbb{N}$, from which we get $q_{n} \in \overline{A a A}$ for all $n \in \mathbb{N}$, and hence:

$$
\overline{\cup_{n \geq 1} A q_{n} A} \subseteq \overline{A a A} .
$$

Let $\varepsilon>0$. Since $\left\langle(a-\varepsilon)_{+}\right\rangle \ll\langle a\rangle$ in $C u(A)=W(A)$ (take into account the fact that $A$ is stable and see [5]), and $\langle a\rangle=\sup \left\langle q_{n}\right\rangle$ (see (10)) we deduce (by definition of the $\ll$ relation) that:

$$
\left\langle(a-\varepsilon)_{+}\right\rangle \leq\left\langle q_{m}\right\rangle
$$

for some $m \in \mathbb{N}$, which implies that $(a-\varepsilon)_{+} \in \overline{A q_{m} A} \subseteq \overline{\cup_{n \geq 1} A q_{n} A}$. Since $\varepsilon>0$ is arbitrary, we have that $a=\lim _{\varepsilon \rightarrow 0}(a-\varepsilon)_{+} \in \overline{\cup_{n \geq 1} A q_{n} A}$, and therefore:

$$
\overline{A a A} \subseteq \overline{\cup_{n \geq 1} A q_{n} A}\left(=\overline{\cup_{n \geq 1} \overline{A q_{n} A}}\right) .
$$

Observe that (12) and (13) prove the equality (11). Hence, if we define $I_{n}:=\overline{A q_{n} A}$ for every $n \in \mathbb{N}$, we have that $\overline{A a A}=\overline{\cup_{n \geq 1} I_{n}}$, where $\left\{I_{n}\right\}$ is an increasing sequence of ideals of $A$ and each $I_{n}$ is generated (as an ideal) by a single projection (namely $q_{n}$ ).

(iv) $\Rightarrow$ (i) Assume (iv). Let $I$ be an ideal of $A$ and let $a \in I^{+}$. Then, by (iv) we have that $\overline{A a A}=\overline{\cup_{n \geq 1} I_{n}}$, where $\left\{I_{n}\right\}$ is an increasing sequence of ideals of $A$ and each $I_{n}$ is generated (as an ideal) by a single projection. This implies that $a$ belongs to the ideal of $A$ generated by $\mathcal{P}(\overline{A a A}) \subseteq \mathcal{P}(I)$, and hence $a$ belongs to the ideal of $A$ generated by $\mathcal{P}(I)$. Since $a \in I^{+}$was arbitrary and each element of $I$ is a linear combination of four positive elements of $I$, it follows that $I$ is generated (as an ideal) by $\mathcal{P}(I)$. But $I$ was an arbitrary ideal of $A$, so we deduce that $A$ has the ideal property.

Remark 2.8. (i) Note that if $A$ is a purely infinite $C^{*}$-algebra, then $W(A)$ has the Riesz interpolation property. The same conclusion holds for the semigroup $V(A)$ consisting of the Murray-von Neumann equivalence classes $[p]$ of projections in $M_{\infty}(A)$. Indeed, let $a_{i}, b_{i} \in M_{\infty}(A)^{+}$be such that $\left\langle a_{i}\right\rangle \leq\left\langle b_{j}\right\rangle, 1 \leq i, j \leq 2$ (in $W(A)$ ). We may assume that $a_{i}, b_{i} \in A^{+}, 1 \leq i \leq 2$. Then, for all $i, j$

$$
\left\langle a_{i}\right\rangle \leq\left\langle a_{1}+a_{2}\right\rangle \leq\left\langle a_{1}\right\rangle+\left\langle a_{2}\right\rangle \leq 2\left\langle b_{j}\right\rangle \leq\left\langle b_{j}\right\rangle .
$$

(We have used here that every non-zero positive element is properly infinite.) 
(ii) For a $C^{*}$-algebra $A$, denote by

$W_{p i}(A)=\left\{\langle a\rangle \in W(A) \mid a=0\right.$ or else properly infinite in $\left.M_{\infty}(A)\right\}$.

Then the same argument as in (i) shows that $W_{p i}(A)$ is a subsemigroup of $W(A)$ with Riesz interpolation. With this language, $[\mathbf{1 7}$, Theorem 4.16] can be rephrased by saying that $A$ is purely infinite if and only if $W(A)=W_{p i}(A)$.

\section{Comparison of positive elements and the ideal property}

We will introduce a notion of comparison of positive elements for unital $C^{*}$-algebras that have (normalized) quasitraces. We will prove that large classes of $C^{*}$-algebras with the ideal property have this comparison property.

We begin recalling some definitions, notation and results. The notion of dimension function was introduced by Cuntz in [6]. A dimension function on a $C^{*}$-algebra $A$ is an additive order preserving function $d: W(A) \rightarrow[0, \infty]$. We can also regard $d$ as a function $M_{\infty}(A)^{+} \rightarrow[0, \infty]$ that respects the rules $d(a \oplus b)=d(a)+d(b)$ and $a \precsim b \Rightarrow d(a) \leq d(b)$ for all $a, b \in M_{\infty}(A)^{+}$. The set of all dimension functions on a $C^{*}$-algebra $A$ will be denoted by $\operatorname{DF}(A)$. A dimension function $d$ on $A$ is said to be lower semicontinuous if $d(a)=\sup _{\varepsilon>0} d\left((a-\varepsilon)_{+}\right)$for all $a \in M_{\infty}(A)^{+}$.

Let $A$ be a unital $C^{*}$-algebra. A (normalized) quasitrace on $A$ is a function $\tau: A \rightarrow \mathbb{C}$ satisfying:

(i) $\tau(1)=1$,

(ii) $0 \leq \tau\left(x x^{*}\right)=\tau\left(x^{*} x\right)$, for all $x \in A$,

(iii) $\tau(a+i b)=\tau(a)+i \tau(b)$, for all $a, b \in A_{s a}$,

(iv) $\tau$ is linear on abelian sub- $C^{*}$-algebras of $A$,

(v) $\tau$ extends to a function from $M_{n}(A)$ to $\mathbb{C}$ satisfying (i)-(iv).

The set of all (normalized) quasitraces on $A$ will be denoted $Q T(A)$. This notion was introduced in $[\mathbf{3}]$. Given $\tau \in Q T(A)$ one may define a $\operatorname{map} d_{\tau}: M_{\infty}(A)^{+} \rightarrow[0, \infty]$ by:

$$
d_{\tau}(a)=\lim _{n \rightarrow \infty} \tau\left(a^{1 / n}\right) .
$$

Note that in fact $d_{\tau}$ takes only real values: $d_{\tau}\left(M_{\infty}(A)^{+}\right) \subseteq[0, \infty)$. Blackadar and Handelman showed in $[\mathbf{3}]$ that $d_{\tau}$ is a lower semicontinuous dimension function on $A$. Note that for all $p \in \mathcal{P}\left(M_{\infty}(A)\right)$ we have that $d_{\tau}(p)=\tau(p)$. 
In the results below we shall need to compare elements of the Cuntz semigroup by means of their values on states. The most common notion that captures this idea is that of strict comparison. Namely, a $C^{*}$-algebra $A$ has strict comparison provided that $a \precsim b$ (for $a, b \in M_{\infty}(A)^{+}$) whenever $d_{\tau}(a)<d_{\tau}(b)$ for every quasitrace $\tau$. For our purposes we need a weakening of this concept, as follows.

Definition 3.1. A unital $C^{*}$-algebra $A$ such that $Q T(A) \neq \emptyset$ is said to have weak strict comparison if it has the property that $a \precsim b$ whenever $a, b \in M_{\infty}(A)^{+}$satisfy the inequality $d(a)<d(b)$ for every $d \in E \cup\{f \in$ $D F(A) \backslash E: f(b)=1\}$, where $E:=\left\{d_{\tau}: \tau \in Q T(A)\right\}$.

Definition 3.2. A unital $C^{*}$-algebra $A$ such that $Q T(A) \neq \emptyset$ is said to have strict comparison of projections if $p \precsim q$ whenever $p, q \in \mathcal{P}\left(M_{\infty}(A)\right)$ satisfy the inequality $\tau(p)<\tau(q)$ for every $\tau \in Q T(A)$.

Theorem 3.3. Let $A$ be a unital $C^{*}$-algebra with the ideal property. Assume moreover that $A$ has strict comparison of projections and that it has finitely many extremal quasitraces. Let $a, b \in M_{\infty}(A)^{+}$such that:

$$
d_{\tau}(a)<d_{\tau}(b), \text { for all } \tau \in Q T(A) .
$$

Then, for every $\varepsilon>0$, there is $m \in \mathbb{N}$ such that $(a-\varepsilon)_{+} \precsim b \otimes 1_{m}$.

Proof: We may assume, without loss of generality, that $a, b \in A^{+}$. Let $\tau_{1}, \tau_{2}, \ldots, \tau_{l}$ be the extremal quasitraces of $A$, for some $l \in \mathbb{N}$. (Hence, each quasitrace of $A$ is a convex combination of $\tau_{i}, 1 \leq i \leq l$.) Since $d_{\tau_{i}}$ is a lower semicontinuous dimension function on $A$, we have that $d_{\tau_{i}}(b)=\sup _{\delta>0} d_{\tau_{i}}\left((b-\delta)_{+}\right), 1 \leq i \leq l$. Hence, $d_{\tau_{i}}(a)<d_{\tau_{i}}(b)$ implies that there exists $\varepsilon_{i}>0$ such that:

$$
d_{\tau_{i}}(a)<d_{\tau_{i}}\left(\left(b-\varepsilon_{i}\right)_{+}\right), \quad 1 \leq i \leq l .
$$

Choose $\varepsilon_{0}>0$ such that $\varepsilon_{0} \leq \varepsilon_{i}, 1 \leq i \leq l$. Since $0<\delta_{1} \leq \delta_{2}$ implies $\left(b-\delta_{2}\right)_{+} \leq\left(b-\delta_{1}\right)_{+}$and hence $\left(b-\delta_{2}\right)_{+} \precsim\left(b-\delta_{1}\right)_{+}$from which one obtains $d_{\tau}\left(\left(b-\delta_{2}\right)_{+}\right) \leq d_{\tau}\left(\left(b-\delta_{1}\right)_{+}\right)$, for every $\tau \in Q T(A)$, the above inequalities imply that:

$$
d_{\tau_{i}}(a)<d_{\tau_{i}}\left(\left(b-\varepsilon_{0}\right)_{+}\right), \quad 1 \leq i \leq l .
$$

On the other hand, since $A$ has the ideal property, Lemma 2.3 implies that for $\varepsilon_{0}>0$ there exist projections $p$ and $q$ in $\mathcal{P}\left(M_{\infty}(A)\right)$ such that $p$ is a finite direct sum of projections of $\overline{A a A}, q$ is a finite direct sum of 
projections of $\overline{A b A}$ and there is some $m \in \mathbb{N}$ such that:

$$
\begin{aligned}
\left(a-\varepsilon_{0}\right)_{+} & \precsim p \\
p & \precsim a \otimes 1_{m} \\
\left(b-\varepsilon_{0}\right)_{+} & \precsim q .
\end{aligned}
$$

Using (14), (16) and (17), we get:

$$
\begin{aligned}
\tau_{i}(p) & =d_{\tau_{i}}(p) \leq d_{\tau_{i}}\left(a \otimes 1_{m}\right)=m d_{\tau_{i}}(a)<m d_{\tau_{i}}\left(\left(b-\varepsilon_{0}\right)_{+}\right) \\
& =d_{\tau_{i}}\left(\left(b-\varepsilon_{0}\right)_{+} \otimes 1_{m}\right) \leq d_{\tau_{i}}\left(q \otimes 1_{m}\right)=\tau_{i}\left(q \otimes 1_{m}\right), \quad 1 \leq i \leq l
\end{aligned}
$$

which obviously implies that:

$$
\tau_{i}(p)<\tau_{i}\left(q \otimes 1_{m}\right), \quad 1 \leq i \leq l .
$$

Multiplying each of these $l$ inequalities with appropriate positive numbers and then summing up all the inequalities, we have that:

$$
\tau(p)<\tau\left(q \otimes 1_{m}\right), \text { for all } \tau \in Q T(A)
$$

(of course, we used here the fact that $\tau_{1}, \tau_{2}, \ldots, \tau_{l}$ are the extremal quasitraces of $A$ ).

Since $A$ has strict comparison of projections, (18) implies that:

$$
p \precsim q \otimes 1_{m} .
$$

Using again condition (ii) in Lemma 2.3 and the fact that $q$ is a finite direct sum of projections of $\overline{A b A}$, it follows that:

$$
q \precsim b \otimes 1_{n}
$$

for some $n \in \mathbb{N}$.

Using (15), (19) and (20) we obtain:

$$
\left(a-\varepsilon_{0}\right)_{+} \precsim p \precsim q \otimes 1_{m} \precsim b \otimes 1_{m n}
$$

which implies that:

$$
\left(a-\varepsilon_{0}\right)_{+} \precsim b \otimes 1_{m n} .
$$

Observe now that for all $0<\varepsilon<\varepsilon_{0}$, we have $\left(b-\varepsilon_{0}\right)_{+} \leq(b-\varepsilon)_{+}$which implies that $\left(b-\varepsilon_{0}\right)_{+} \precsim(b-\varepsilon)_{+}$and hence $d_{\tau}\left(\left(b-\varepsilon_{0}\right)_{+}\right) \leq d_{\tau}\left((b-\varepsilon)_{+}\right)$, for all $\tau \in Q T(A)$. Using this fact and (14), we get:

$$
d_{\tau_{i}}(a)<d_{\tau_{i}}\left((b-\varepsilon)_{+}\right) \text {, for all } 0<\varepsilon<\varepsilon_{0}, \quad 1 \leq i \leq l .
$$

Working as above, we obtain that for each $0<\varepsilon<\varepsilon_{0}$ there exists $k=k(\varepsilon) \in \mathbb{N}$ such that:

$$
(a-\varepsilon)_{+} \precsim b \otimes 1_{k} .
$$


Finally, for every $\varepsilon>\varepsilon_{0}$ we have $(a-\varepsilon)_{+} \leq\left(a-\varepsilon_{0}\right)_{+}$, which implies that $(a-\varepsilon)_{+} \precsim\left(a-\varepsilon_{0}\right)_{+}$, and hence, using also (21), we obtain:

$$
(a-\varepsilon)_{+} \precsim b \otimes 1_{m n} .
$$

This ends the proof.

Remark 3.4. For all $a$ and $b \in A^{+}$, the conclusion of the above result that, for every $\varepsilon>0$, there is $m$ such that $(a-\varepsilon)_{+} \precsim b \otimes 1_{m}$ can be rephrased by saying that $a \in \overline{A b A}$, as mentioned in [26, comment before Lemma 4.1]. We shall be using this below.

If $A$ is a unital $C^{*}$-algebra, we will denote by $T(A)$ the set of tracial states of $A$.

Corollary 3.5. Let $A$ be a unital AH algebra with the ideal property and with finitely many extremal tracial states, and let $a, b \in M_{\infty}(A)^{+}$ such that $d_{\tau}(a)<d_{\tau}(b)$, for all $\tau \in T(A)$. Then, for every $\varepsilon>0$ there is $m \in \mathbb{N}$ such that $(a-\varepsilon)_{+} \precsim b \otimes 1_{m}$.

Proof: Let $U$ be an arbitrary $U H F$ algebra, that is, $U$ is the inductive limit of a sequence $\left\{M_{n(k)}\right\}_{k}$ of matrix algebras via unital *-homomorphisms $M_{n(k)} \rightarrow M_{n(k+1)}$. Define $B:=A \otimes U$. Then, clearly, $B$ is a unital $A H$ algebra with the ideal property and with slow dimension growth (in the sense of Gong [11]). Then, since $B$ is a unital exact $C^{*}$-algebra, by a theorem of Haagerup [15] we have that $Q T(B)=T(B)$. Hence, by $[\mathbf{1 8}$, Theorem $5.1(\mathrm{~b})]$ it follows that $B$ has strict comparison of projections. Let $T(U)=\{\sigma\}$. Then, we have that $T(B)=T(A \otimes U)=\{\tau \otimes \sigma: \tau \in T(A)\}$. Therefore, clearly, $B=A \otimes U$ has finitely many extremal tracial states, since $A$ has finitely many extremal tracial states. We may assume, without any loss of generality, that $a, b \in A^{+}$. Define $\widetilde{a}:=a \otimes 1, \widetilde{b}:=b \otimes 1 \in(A \otimes U)^{+}=B^{+}$. Then, for all $\rho=\tau \otimes \sigma \in T(B)(\tau \in T(A)))$, we have by hypothesis:

$$
d_{\rho}(\widetilde{a})=d_{\tau}(a)<d_{\tau}(b)=d_{\rho}(\widetilde{b}) .
$$

Using now Theorem 3.3 and Remark 3.4 we deduce that $a \otimes 1=\widetilde{a} \in$ $\overline{B \widetilde{b} B}=\overline{(A \otimes U)(b \otimes 1)(A \otimes U)}$, from which we easily conclude (using, e.g., Fubini maps) that $a \in \overline{A b A}$, which implies the conclusion, by $[\mathbf{1 7}$, Proposition 2.7(v)].

The next two theorems are the main results of this section. Recall first that a positive ordered abelian semigroup $W$ (in particular, the Cuntz semigroup of a $C^{*}$-algebra) is said to be almost unperforated if for all $x, y \in W$ and all $m, n \in \mathbb{N}$, with $n x \leq m y$ and $n>m$, one has $x \leq y$ (see, e.g. [26]). 
Theorem 3.6. Let $A$ be a unital $C^{*}$-algebra with the ideal property. Assume moreover that $A$ has strict comparison of projections and finitely many extremal quasitraces and that $W(A)$ is almost unperforated. Then $A$ has weak strict comparison.

Proof: Use Theorem 3.3, Remark 3.4 and [26, Corollary 4.7].

Theorem 3.7. Let $A$ be a unital $A H$ algebra with the ideal property and with finitely many extremal tracial states and such that $W(A)$ is almost unperforated. Then A has weak strict comparison.

Proof: Use Corollary 3.5, Remark 3.4 and [26, Corollary 4.7].

Theorem 3.8. Let $A$ be a unital $A H$ algebra with the ideal property and with finitely many extremal tracial states and let $B$ be a unital, simple, infinite dimensional $A H$ algebra with no dimension growth and with a unique tracial state. Then $A \otimes B$ has weak strict comparison.

Proof: Observe first that since both $A$ and $B$ have the ideal property and $A$ (or $B$ ) is exact, it follows that $A \otimes B$ has the ideal property (use, e.g., $[\mathbf{2 0}$, Corollary 1.3]). On the other hand, by a result in $[\mathbf{3 0}], B$ is $\mathcal{Z}$-stable, that is $B \cong B \otimes \mathcal{Z}$, where $\mathcal{Z}$ is the Jiang-Su algebra [16]. Hence the unital $A H$ algebra with the ideal property $A \otimes B$ is $\mathcal{Z}$-stable, i.e. $A \otimes B \cong(A \otimes B) \otimes \mathcal{Z}$, and then [26, Theorem 4.5] implies that $W(A \otimes B)$ is almost unperforated. Note that if $T(B)=\{\sigma\}$, then $T(A \otimes B)=\{\tau \otimes \sigma: \tau \in T(A)\}$ and since $A$ has finitely many extremal tracial states, it is obvious that $A \otimes B$ has also finitely many extremal tracial states. Now, the fact that $A \otimes B$ has weak strict comparison follows from Theorem 3.7.

Remark 3.9. We may say that a unital $C^{*}$-algebra $A$ with $Q T(A) \neq \emptyset$ has almost weak strict comparison if $A$ satisfies all the conditions in the definition of weak strict comparison (see Definition 3.1) with the only difference that the condition:

$$
d(a)<d(b) \text { for all } d \in E
$$

is replaced by the new condition:

(**) there is $\varepsilon_{0}>0$ such that $d(a)<d\left(\left(b-\varepsilon_{0}\right)_{+}\right)$for all $d \in E$,

with $E$ as in Definition 3.1 above (of course, we still request that $d(a)<$ $d(b)$ for every $d \in\{f \in D F(A) \backslash E: f(b)=1\})$. 
In the proof of Theorem 3.3 we showed, in particular, that in the case when a unital $C^{*}$-algebra $A$ has finitely many extremal quasitraces, then $(*) \Longrightarrow(* *)$. Therefore, in this case, if $A$ has almost weak strict comparison, it follows that $A$ has weak strict comparison. Note that if we drop the condition that the $C^{*}$-algebra $A$ has finitely many extremal quasitraces (tracial states), the conclusions of Theorem 3.3 and of Corollary 3.5 remain true if we replace in their hypotheses condition $(*)$ by condition $(* *)$ as above. Also, it is easy to see that, if in Theorems 3.6, 3.7, 3.8 we drop the condition that $A$ has finitely many extremal quasitraces (tracial states) and the condition that $\mathrm{B}$ has a unique tracial state (in Theorem 3.8), then they remain true if we replace in their conclusions "weak strict comparison" by "almost weak strict comparison" (to show these results we use the same proofs). In conclusion, we thus obtain generalizations of all the results proved in Section 3.

\section{Acknowledgements}

The second named author was partially supported by a MICINN grant (Spain) through Project MTM2011-28992-C02-01, and by the Comissionat per Universitats i Recerca de la Generalitat de Catalunya (SGR20091389). Part of this work was carried out during the AIM Workshop "The Cuntz semigroup", held in November 2009. The authors are grateful to AIM and its staff for their support and working conditions provided. It is also a pleasure to thank Aaron Tikuisis for comments on a first draft that allowed us to simplify some arguments and led to an improved exposition.

\section{References}

[1] R. Antoine, J. Bosa, And F. Perera, Completions of monoids with applications to the Cuntz semigroup, Internat. J. Math. 22(6) (2011), 837-861. DOI: 10.1142/S0129167X11007057.

[2] P. Ara, F. Perera, and A. S. Toms, $K$-theory for operator algebras. Classification of $C^{*}$-algebras, in: "Aspects of operator algebras and applications", Contemp. Math. 534, Amer. Math. Soc., Providence, RI, 2011, pp. 1-71. DOI : 10.1090/conm/534/10521.

[3] B. Blackadar and D. Handelman, Dimension functions and traces on $C^{*}$-algebras, J. Funct. Anal. 45(3) (1982), 297-340. DOI: 10.1016/0022-1236(82) 90009-X. 
[4] L. G. Brown and G. K. Pedersen, $C^{*}$-algebras of real rank zero, J. Funct. Anal. 99(1) (1991), 131-149. DOI: 10.1016/0022-1236 (91) 90056-B.

[5] K. T. Coward, G. A. Elliott, and C. Ivanescu, The Cuntz semigroup as an invariant for $C^{*}$-algebras, J. Reine Angew. Math. 623 (2008), 161-193. DOI: 10.1515/CRELLE. 2008.075.

[6] J. Cuntz, Dimension functions on simple $C^{*}$-algebras, Math. Ann. 233(2) (1978), 145-153. DOI: 10.1007/BF01421922.

[7] J. Cuntz, $K$-theory for certain $C^{*}$-algebras, Ann. of Math. (2) 113(1) (1981), 181-197. DOI: 10.2307/1971137.

[8] M. DĂDĂRLAT, Reduction to dimension three of local spectra of real rank zero $C^{*}$-algebras, J. Reine Angew. Math. 460 (1995), 189-212. DOI : $10.1515 /$ crll.1995.460.189.

[9] G. A. Elliott, The classification problem for amenable $C^{*}$-algebras, in: "Proceedings of the International Congress of Mathematicians", Vol. 1, 2 (Zürich, 1994), Birkhäuser, Basel, 1995, pp. $922-932$.

[10] G. A. Elliott And A. S. Toms, Regularity properties in the classification program for separable amenable $C^{*}$-algebras, Bull. Amer. Math. Soc. (N.S.) 45(2) (2008), 229-245. DOI: 10.1090/S02730979-08-01199-3.

[11] G. Gong, On inductive limits of matrix algebras over higherdimensional spaces. II., Math. Scand. 80(1) (1997), 56-100.

[12] G. Gong, On the classification of simple inductive limit $C^{*}$-algebras. I. The reduction theorem, Doc. Math. 7 (2002), 255-461 (electronic).

[13] G. Gong, C. Jiang, L. Li, and C. Pasnicu, $A \mathbb{T}$ structure of $A H$ algebras with the ideal property and torsion free $K$-theory, J. Funct. Anal. 258(6) (2010), 2119-2143. DOI: 10.1016/j.jfa.2009.11.016.

[14] G. Gong, C. Jiang, L. Li, and C. Pasnicu, A reduction theorem for $A H$ algebras with the ideal property, Preprint.

[15] U. HaAgerup, Every quasi-trace on an exact $C^{*}$-algebra is a trace, Preprint (1991).

[16] X. Jiang And H. Su, On a simple unital projectionless $C^{*}$-algebra, Amer. J. Math. 121(2) (1999), 359-413. DOI: 10.1353/ajm.1999. 0012.

[17] E. Kirchberg and M. Rørdam, Non-simple purely infinite $C^{*}$-algebras, Amer. J. Math. 122(3) (2000), 637-666. DOI: 10.1353/ajm. 2000.0021 . 
[18] C. Pasnicu, Shape equivalence, nonstable $K$-theory and $A H$ algebras, Pacific J. Math. 192(1) (2000), 159-182. DOI: 10.2140/ pjm.2000.192.159.

[19] C. Pasnicu, On the $A H$ algebras with the ideal property, J. Operator Theory 43(2) (2000), 389-407.

[20] C. Pasnicu And M. RøRdam, Tensor products of $C^{*}$-algebras with the ideal property, J. Funct. Anal. 177(1) (2000), 130-137. DOI : $10.1006 / \mathrm{jfan} .2000 .3630$.

[21] C. Pasnicu And M. Rørdam, Purely infinite $C^{*}$-algebras of real rank zero, J. Reine Angew. Math. 613 (2007), 51-73. DOI: 10.1515/CRELLE. 2007.091.

[22] F. PERERA, The structure of positive elements for $C^{*}$-algebras with real rank zero, Internat. J. Math. 8(3) (1997), 383-405. DOI: 10.1142/S0129167X97000196.

[23] M. RøRDAM, On the structure of simple $C^{*}$-algebras tensored with a UHF-algebra. II, J. Funct. Anal. 107(2) (1992), 255-269. DOI: 10.1016/0022-1236(92) 90106-S.

[24] M. RøRDAM, Classification of nuclear, simple $C^{*}$-algebras, in: "Classification of nuclear $C^{*}$-algebras. Entropy in operator algebras", Encyclopaedia Math. Sci. 126, Springer, Berlin, 2002, pp. $1-145$.

[25] M. RøRDAM, A simple $C^{*}$-algebra with a finite and an infinite projection, Acta Math. 191(1) (2003), 109-142. DOI: 10.1007/ BF02392697.

[26] M. RøRDAM, The stable and the real rank of $\mathcal{Z}$-absorbing $C^{*}$-algebras, Internat. J. Math. 15(10) (2004), 1065-1084. DOI : 10.1142/ S0129167X04002661.

[27] A. Tikuisis, The Cuntz semigroup of continuous functions into certain simple $C^{*}$-algebras, Internat. J. Math. 22(8) (2011), 1051-1087. DOI: 10.1142/S0129167X11007136.

[28] A. Toms, On the independence of $K$-theory and stable rank for simple $C^{*}$-algebras, J. Reine Angew. Math. 578 (2005), 185-199. DOI: $10.1515 / \operatorname{crll} .2005 .2005 .578 .185$.

[29] A. S. Toms, On the classification problem for nuclear $C^{*}$-algebras, Ann. of Math. (2) 167(3) (2008), 1029-1044. DOI: 10.4007/ annals. 2008.167.1029.

[30] A. S. Toms And W. Winter, $\mathcal{Z}$-stable ASH algebras, Canad. J. Math. 60(3) (2008), 703-720. DOI: 10.4153/CJM-2008-031-6. 
Cornel Pasnicu:

Department of Mathematics

The University of Texas at San Antonio

San Antonio, TX 78249

USA

E-mail address: Cornel.Pasnicu@utsa.edu

Francesc Perera:

Departament de Matemàtiques

Universitat Autònoma de Barcelona

08193 Bellaterra (Barcelona)

Spain

E-mail address: perera@mat.uab.cat

Primera versió rebuda el 30 de març de 2012, darrera versió rebuda el 28 de setembre de 2012 . 